\title{
Early-life antibiotic use and risk of asthma and eczema: results of a discordant twin study
}

\author{
Elise M.A. Slob (10 ${ }^{1,2}$, Bronwyn K. Brew ${ }^{3,4}$, Susanne J.H. Vijverberg ${ }^{1,2}$, \\ Chantal J.A.R. Kats ${ }^{1}$, Cristina Longo ${ }^{1}$, Mariëlle W. Pijnenburg ${ }^{5}$, \\ Toos C.E.M. van Beijsterveldt ${ }^{6}$, Conor V. Dolan $^{6}$, Meike Bartels $^{6}$, \\ Patrick Magnusson ${ }^{3}$, Paul Lichtenstein ${ }^{3}$, Tong Gong ${ }^{3}$, Gerard H. Koppelman (1) 7,8, \\ Catarina Almqvist ${ }^{3,9}$, Dorret I. Boomsma $\mathbb{0}^{6}$ and Anke H. Maitland-van der Zee ${ }^{1,2}$
}

@ERSpublications

Large twin studies show that antibiotics in early life are associated with risk of asthma, regardless of familial environment and genetics, and possibly due to early infections. Risks and benefits of antibiotics use in infants should be considered. http://bit.ly/372Qu5A

Cite this article as: Slob EMA, Brew BK, Vijverberg SJH, et al. Early-life antibiotic use and risk of asthma and eczema: results of a discordant twin study. Eur Respir J 2020; 55: 1902021 [https://doi.org/10.1183/ 13993003.02021-2019].

\section{ABSTRACT}

Rationale: Early-life antibiotic use has been associated with the development of atopic diseases, but the aetiology remains unclear. To elucidate the aetiology, we used a discordant twin design to control for genetic and environmental confounding.

Methods: We conducted a retrospective cohort study in twins aged 3-10 years from the Netherlands Twin Register (NTR, $\mathrm{n}=35365$ ) and a replication study in twins aged 9 years from the Childhood and Adolescent Twin Study in Sweden (CATSS, $\mathrm{n}=7916$ ). Antibiotic use was recorded at age $0-2$ years. Doctor-diagnosed asthma and eczema were reported by parents when children were aged 3-12 years in both cohorts. Individuals were included in unmatched analyses and in co-twin control analyses with disease discordant twin pairs.

Results: Early-life antibiotic use was associated with increased risk of asthma (NTR OR 1.34, 95\% CI 1.281.41; CATSS OR 1.45, 95\% CI 1.34-1.56) and eczema (NTR OR 1.08, 95\% CI 1.03-1.13; CATSS OR 1.07, 95\% CI 1.01-1.14) in unmatched analyses. Co-twin analyses in monozygotic and dizygotic twin pairs showed similar results for asthma (NTR OR 1.54, 95\% CI 1.20-1.98; CATSS OR 2.00, 95\% CI 1.28-3.13), but opposing results for eczema in the NTR (OR 0.99, 95\% CI 0.80-1.25) and the CATSS (OR 1.67, 95\% CI 1.12-2.49). The risk of asthma increased for antibiotics prescribed for respiratory infections (CATSS OR 1.45, 95\% CI 1.34-1.56), but not for antibiotics commonly used for urinary tract/skin infections (CATSS OR 1.02, 95\% CI 0.88-1.17).

Conclusion: Children exposed to early-life antibiotic use, particularly prescribed for respiratory infections, may be at higher risk of asthma. This risk can still be observed when correcting for genetic and environmental factors. Our results could not elucidate whether the relationship between early-life antibiotic use and eczema is confounded by familial and genetic factors. 


\section{Introduction}

The worldwide prevalence of atopic diseases increased over the last decades, having social and economic impacts on society [1,2]. The atopic disease epidemic parallels increased antibiotics use [3]. A recent study reported that antibiotics are prescribed to Dutch children for one in four infectious episodes diagnosed by a general practitioner (714 prescriptions per 1000 patient-years in 1-year-olds) [4].

Different hypotheses have been put forward to explain the relationship between asthma/eczema development and early-life antibiotic use. First, antibiotics perturb the gastrointestinal tract microbiota and promote a shift from a T-helper cell (Th) 1 to a Th2 response [5], increasing the risk of atopic diseases [6]. Antibiotics change the microbiota, with broad-spectrum antibiotics thought to provide more changes than narrow-spectrum antibiotics and subsequently increasing the susceptibility of developing asthma. Early studies investigating microbiota differences in children with asthma and eczema support this hypothesis [7-9]. Second, undiagnosed asthma may be associated with increased susceptibility to infections and subsequent antibiotic treatment or early-onset symptoms of asthma may have been misdiagnosed and treated with antibiotics (a phenomenon known as reverse causation) [10]. Furthermore, the association between antibiotics and asthma may be confounded by a third factor such as respiratory tract infections, which are an indication for antibiotics and might also increase the risk of developing asthma later in life [10-13]. Nevertheless, recent evidence suggests no association between respiratory syncytial virus prevention in late pre-terms and incident asthma at school age [14]. Conflicting results have been published from epidemiological studies attempting to investigate the relationship between asthma and respiratory infections [15]. Lastly, unmeasured confounding factors such as environmental and genetic factors shared within families (lifestyle, health-seeking behaviour or genetic predisposition to respiratory infection) may have led to a spurious positive relationship between antibiotics and asthma.

Previous studies suggested that early-life antibiotic use is associated with increased risk of asthma or eczema, but results were inconsistent [16-19]. Two meta-analyses concluded that exposure to antibiotics in the first 2 years of life increased the risk of both asthma (OR 2.18,95\% CI 1.04-4.60; four studies with a total of 8284 patients) and eczema (OR 1.26, 95\% CI 1.15-1.37; 22 studies with a total of 394517 patients) $[20,21]$. A large population-based study with sibling comparisons suggested that the association between early-life antibiotic exposure and subsequent childhood asthma may be confounded by shared familial factors, in addition to confounding by indication [12]. While the strength of the sibling design is the inherent control for shared familial factors, some additional genetic confounding may remain as siblings only share half of their genes. Monozygotic twins share the same genes and environment, and therefore a twin study is an improvement on the sibling design. Furthermore, the role of shared familial factors is unknown for the association between antibiotics and eczema [22].

We aimed to assess whether early-life antibiotic use is associated with asthma and eczema in Dutch (discovery cohort) and Swedish (replication cohort) monozygotic and dizygotic twin pairs. A twin study design including twin pairs discordant for eczema/asthma provides a unique opportunity to study the impact of antibiotic use on asthma/eczema development while controlling for shared familial environment and genetic factors.

\section{Methods}

\section{Study design and populations}

We conducted a retrospective cohort study in twins aged 3-12 years from the Netherlands Twin Registry (NTR) and replicated our results in twins aged 9 years from the Childhood and Adolescent Twin Study in

Affiliations: 'Dept of Respiratory Medicine, Amsterdam University Medical Center, University of Amsterdam, Amsterdam, The Netherlands. ${ }^{2}$ Dept of Paediatric Pulmonology, Amsterdam University Medical Center, University of Amsterdam, Amsterdam, The Netherlands. ${ }^{3}$ Dept of Medical Epidemiology and Biostatistics, Karolinska Institutet, Stockholm, Sweden. ${ }^{4}$ National Perinatal Epidemiology and Statistics Unit, Centre for Big Data Research in Health and Dept of Women's and Children's Health, University of New South Wales, Sydney, Australia. ${ }^{5}$ Dept of Paediatrics, Division of Respiratory Medicine and Allergology, ErasmusMC, University Medical Center Rotterdam, Rotterdam, The Netherlands. ${ }^{6}$ Netherlands Twin Register, Dept of Biological Psychology, Vrije Universiteit Amsterdam, Amsterdam, The Netherlands. ${ }^{7}$ Dept of Paediatric Pulmonology and Paediatric Allergology, Beatrix Children's Hospital, University of Groningen, University Medical Center Groningen, Groningen, The Netherlands. ${ }^{8}$ Groningen Research Institute for Asthma and COPD (GRIAC), University of Groningen, University Medical Center Groningen, Groningen, The Netherlands. ${ }^{9}$ Paediatric Allergy and Pulmonology Unit, Astrid Lindgren Children's Hospital, Karolinska University Hospital, Stockholm, Sweden.

Correspondence: Anke H. Maitland-van der Zee, Dept of Respiratory Medicine, Amsterdam University Medical Center, Meibergdreef 9, 1100 DE Amsterdam, The Netherlands. E-mail: a.h.maitlanddamsterdamumc.nl 
Sweden (CATSS). Children were excluded if data describing their medication use or asthma/eczema diagnosis were not available. All data were deidentified.

To examine whether early-life antibiotic use was associated with asthma and eczema [23], we undertook three steps. First, we conducted an unmatched cohort analysis to assess whether an association between early-life antibiotic use and asthma or eczema was found. Children with asthma/eczema were compared with healthy control individuals. Controls from twin pairs discordant for asthma or eczema were excluded from this first step, to adjust for twin relatedness. These analyses establish the presence of an association at the population level, without accounting for genetic and/or other shared familial factors. Second, we performed a matched co-twin control analysis in all monozygotic and in same-sex dizygotic twin pairs discordant for either asthma or eczema. In a separate analysis only same-sex dizygotic twin pairs discordant for either asthma or eczema were included. Both analyses adjusted for the contribution of shared familial environment and partially for shared genes. Third, we conducted a matched co-twin control analysis in monozygotic twins discordant for asthma or eczema, which controlled for all shared environmental factors and all confounding by genetic factors. We investigated dizygotic and monozygotic twins separately to compare the role of genetics in the association. If the associations generated by the monozygotic twins are stronger than by the dizygotic twins, it could imply that at least part of the association is driven by genes.

\section{Discovery cohort}

The NTR is a birth cohort which was initiated in 1987 [24]. Twins are registered by their parents after birth, and recruited with the help of a commercial organisation in the Netherlands called Felicitas and the Dutch Association for Parents of Multiple Births. Data are collected by sending surveys at ages 0, 2, 3, $5,7,9 / 10$ and 12 years. The response rate varied from $40 \%$ to $75 \%$. For the current study, data collected between 1989 and 2016 were used.

\section{Replication cohort}

The replication cohort consisted of Swedish twins born between January 2005 and February 2010 in the CATSS [25]. Parents were interviewed about their children's health, including questions about asthma and eczema, via a telephone questionnaire at age 9 years. Twins were identified via the Swedish Twin Registry, including all Swedish twins. The participation rate was 79.5\%. Data were linked to Swedish national health registers by personal identity number via the National Board of Health and Welfare and Statistics in Sweden [26]. The Swedish Prescribed Drug Register, Medical Birth Register and National Patient Register were linked to CATSS information.

\section{Outcomes}

The primary outcomes, i.e. parental-reported ever-asthma and ever-eczema diagnosis, were derived at ages 3, 5, 7 and/or 10 years in Dutch twins (supplementary table S1). Asthma or eczema was considered to be present if reported in at least one survey. In the CATSS, parents of twins were asked at age 9 years whether their child ever had asthma and/or eczema (supplementary table S2).

\section{Exposure}

Exposure to antibiotics was defined between 0 and 2 years of age as any parental-reported antibiotics use in Dutch twins and as any prescription claim for antibiotics (Anatomical Therapeutic Chemical (ATC) codes) in the Swedish Prescribed Drug Register for Swedish twins (supplementary table S2).

\section{Confounders and covariates}

A directed acyclic graph was drawn to select covariates (supplementary figure S1). Data describing sex, delivery mode, birthweight, hours of outside childcare, breastfeeding, and educational attainment of the mother and father were derived from the NTR surveys at ages $0,2,3,5,7$ and 9/10 years. In the CATSS, data describing breastfeeding and hours of outside childcare were not available. Perinatal covariates in the CATSS were obtained from the Medical Birth Register, and educational attainment was obtained by questionnaire and divided in four groups: $\leqslant 9$ years, $10-12$ years, $<2$ years tertiary and $\geqslant 2$ years tertiary.

\section{Statistical analysis}

We used a logistic regression model with a generalised estimating equation approach for unmatched analysis to account for twin relatedness adjusted for all measured covariates. In the co-twin matched analyses, conditional logistic regression models were used within each discordant twin pair to account for shared genetic and/or environmental factors. Analyses were performed using SPSS version 25 (IBM, Armonk, NY, USA). 
To investigate the robustness of the findings, we performed sensitivity analyses where we 1) only assessed the parental-reported diagnosis in Dutch twins aged over 5 years (for asthma) and 3 years (for eczema); 2) used validated definitions for asthma and eczema in the CATSS based on medication from the Swedish Prescribed Drug Register and diagnoses from the National Patient Register (supplementary table S2); 3) only investigated CATSS with parental-reported asthma diagnosis after 2 years of age; 4) stratified by antibiotics commonly prescribed for respiratory infections and urinary tract/skin infections in the CATSS adjusting for other antibiotics prescribed in early life (supplementary table S2) [12]; and 5) stratified by broad-spectrum and narrow-spectrum antibiotics in the CATSS adjusting for other antibiotics prescribed in early life (supplementary table S2) [27].

A sample size of 2480 and 2220 children for asthma and eczema, respectively, would be needed to detect an OR of 1.30, assuming a prevalence of 35\% for antibiotic use in early life, $25 \%$ incidence of asthma and $31 \%$ incidence of eczema (based on our dataset) [28, 29].

\section{Results}

Summary characteristics of the Dutch and Swedish populations are described in table 1 for the asthma analysis and table 2 for the eczema analysis. We included 35365 individuals in the NTR and 7916 in the CATSS; the total numbers of individuals for the asthma and eczema studies slightly differ based on the number of responses to the questions about the asthma and eczema diagnoses. The parental-reported prevalence of an ever-diagnosis of asthma was $15.1 \%$ at age $3-10$ years in the NTR and $17.5 \%$ at age 9 years in the CATSS. For eczema, the prevalences were $19.1 \%$ and $21.9 \%$, respectively. The exposure to at least one prescription of any antibiotic between 0 and 2 years was $34.8 \%$ in the NTR and $44.9 \%$ in the CATSS. The proportion of children born after a gestational age of $\geqslant 37$ weeks was higher in the NTR compared with the CATSS (58.6\% versus $46.1 \%$ ), and higher in children with asthma born at gestational age $<37$ weeks compared with the whole cohort in the NTR $(48.4 \%$ versus $41.4 \%)$ and in the CATSS (61.8\% versus $53.9 \%)$. In the NTR, there were more children with asthma not receiving breastfeeding compared with children without asthma (46.3\% versus $40.2 \%$ ). In the CATSS we did not identify differences in summary characteristics between children with asthma or eczema and children without the disease. Mothers in the CATSS more often delivered via caesarean section compared with mothers in the NTR (41.1\% versus $28.6 \%)$.

\section{Antibiotics and asthma}

Use of any antibiotic was associated with an increased risk of asthma in the NTR (OR 1.34, 95\% CI 1.28-1.41) and the CATSS (OR 1.45, 95\% CI 1.34-1.56) (table 3).

In the dizygotic co-twin control analyses, the risk for developing asthma was associated with exposure to antibiotic use at age $0-2$ years in both cohorts (NTR OR 1.60, 95\% CI 1.18-2.16; CATSS OR 1.86, 95\% CI 1.11-3.18) (table 3). For monozygotic discordant twins, estimates pointed in the same direction, but did not reach statistical significance (NTR OR 1.52, 95\% CI 0.89-2.60; CATSS OR 2.23, 95\% CI 0.90-5.53).

\section{Sensitivity analyses for asthma}

Sensitivity analyses, in which we 1) only included asthma in those aged over 5 years (NTR), 2) used a validated definition for asthma and eczema based on medication and diagnosis (CATSS) and 3) only investigated twins with parental answers to questions on any given asthma diagnosis from 2 years of age (CATSS), demonstrated similar findings (supplementary tables S3-S5).

\section{Antibiotics commonly prescribed for respiratory and urinary tract/skin infections and asthma}

Subgroup analyses showed that asthma risk was associated with early-life exposure to antibiotics commonly prescribed for respiratory tract infections (OR 1.45, 95\% CI 1.34-1.56) (supplementary table S6a). After restricting to dizygotic twins, the risk of developing asthma remained (OR 1.91, 95\% CI 1.12-3.27) (supplementary table S6a). In monozygotic twins, the average effect size remained, but precision was lost (OR 1.82, 95\% CI 0.76-2.37) (supplementary table S6a).

Early-life exposure to antibiotics commonly prescribed for urinary tract/skin infections was, however, not associated with asthma in the unmatched analysis (OR 1.02, 95\% CI 0.88-1.17) (table 4). Results for children with antibiotic use commonly prescribed for urinary tract/skin infections, after controlling for antibiotics commonly prescribed for respiratory tract infections during infancy, could not be interpreted due to insufficient power (supplementary table S6b and S10).

\section{Narrow-spectrum and broad-spectrum antibiotics and asthma}

Unmatched cohort analyses for use of broad-spectrum and narrow-spectrum antibiotics showed that both were associated with increased risk of asthma, with the highest risk associated with narrow-spectrum 
TABLE 1 Summary characteristics of the Netherlands Twin Registry (NTR) and the Childhood and Adolescent Twin Study in Sweden (CATSS) populations in the unmatched cohort study for asthma (step 1)

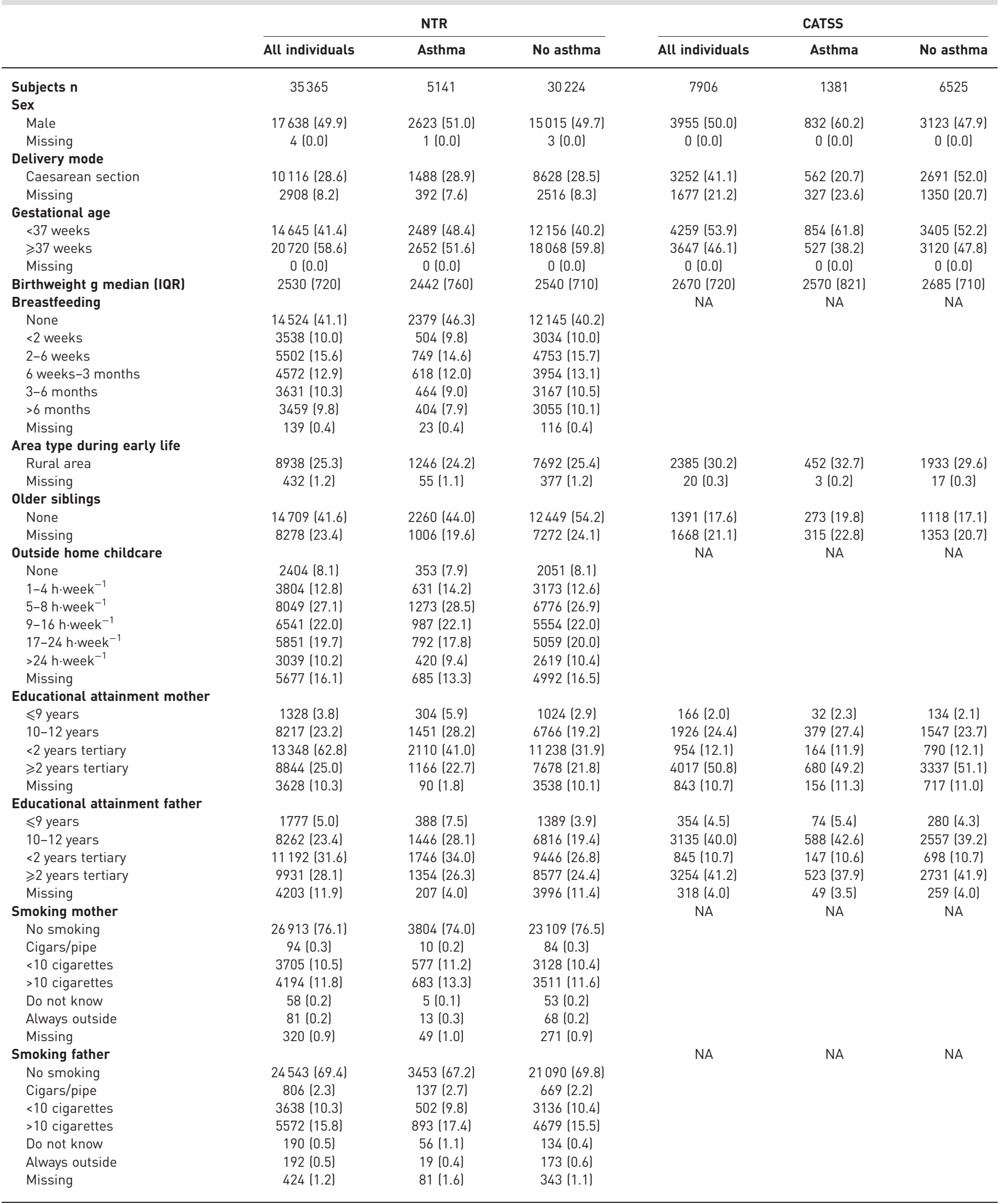

Data are presented as $\mathrm{n}(\%)$, unless otherwise stated. IQR: interquartile range; NA: not available. 
TABLE 2 Summary characteristics of the Netherlands Twin Registry (NTR) and the Childhood and Adolescent Twin Study in Sweden (CATSS) populations in the unmatched cohort study for eczema (step 1)

\begin{tabular}{|c|c|c|c|c|c|c|}
\hline & \multicolumn{3}{|c|}{ NTR } & \multicolumn{3}{|c|}{ CATSS } \\
\hline & All individuals & Eczema & No eczema & All individuals & Eczema & No eczema \\
\hline Subjects $n$ & 35183 & 6479 & 28686 & 7916 & 1730 & 6186 \\
\hline Male & $17520(49.7)$ & $3239(49.9)$ & 14281 (49.8) & $3962(50.1)$ & 809 (46.8) & $3153(51.0)$ \\
\hline Missing & $4(0.0)$ & $1(0.0)$ & $3(0.0)$ & $0(0.0)$ & $0(0.0)$ & $0(0.0)$ \\
\hline \multicolumn{7}{|l|}{ Delivery mode } \\
\hline \multicolumn{7}{|l|}{ Gestational age } \\
\hline$<37$ weeks & $14511(41.2)$ & $2702(41.6)$ & 11809 (41.2) & 4270 (53.9) & 949 (54.9) & $3321(53.7)$ \\
\hline$\geqslant 37$ weeks & $20672(58.8)$ & $3795(58.4)$ & $16877(58.8)$ & $3646(46.1)$ & $781(45.1)$ & $2865(46.3)$ \\
\hline Missing & $0(0.0)$ & $0(0.0)$ & $0(0.0)$ & $0(0.0)$ & $0(0.0)$ & $0(0.0)$ \\
\hline Birthweight g median (IQR) & $2530(720)$ & 2530 (730) & $2530(720)$ & $2670(720)$ & $2655(705)$ & $2670(725)$ \\
\hline Breastfeeding & & & & NA & NA & NA \\
\hline None & $14410(40.1)$ & $2589(39.8)$ & 11821 (41.2) & & & \\
\hline$>6$ months & $3451(9.8)$ & $669(10.3)$ & $2782(9.7)$ & & & \\
\hline Missing & $136(0.4)$ & $22(0.3)$ & $114(0.5)$ & & & \\
\hline \multicolumn{7}{|l|}{ Area type during early life } \\
\hline Rural area & $8897(25.3)$ & $1520(23.4)$ & $7377(25.7)$ & $2392(30.2)$ & 495 (28.7) & $1897(30.7)$ \\
\hline Missing & $434(1.2)$ & $85(1.3)$ & $349(1.2)$ & $22(0.3)$ & $5(0.3)$ & $17(0.3)$ \\
\hline \multicolumn{7}{|l|}{ Older siblings } \\
\hline None & $14670(54.5)$ & $3072(53.8)$ & $11598(54.6)$ & 1389 (22.2) & $357(25.6)$ & $1032(21.2)$ \\
\hline Missing & $8249(23.4)$ & $790(12.2)$ & 7459 (26.0) & 1652 (20.9) & $335(19.4)$ & $1317(21.3)$ \\
\hline Outside home childcare & & & & NA & NA & NA \\
\hline None & $2397(6.8)$ & $394(6.1)$ & 2003 (6.9) & & & \\
\hline $1-4 \mathrm{~h} \cdot$ week $^{-1}$ & $3761(10.7)$ & $689(10.6)$ & 3072 (10.7) & & & \\
\hline $5-8 \mathrm{~h} \cdot$ week $^{-1}$ & $7968(22.6)$ & $1472(22.7)$ & $6496(22.6)$ & & & \\
\hline $9-16 h \cdot$ week $^{-1}$ & $6534(18.6)$ & $1230(18.9)$ & $5304(22.1)$ & & & \\
\hline $17-24$ h.week ${ }^{-1}$ & $5857(16.6)$ & $1142(17.6)$ & 4715 (18.5) & & & \\
\hline \multicolumn{7}{|l|}{ Educational attainment father } \\
\hline$\leqslant 9$ years & $1906(5.4)$ & 345 (5.3) & $1561(0.6)$ & $354(4.5)$ & $60(3.5)$ & $294(4.8)$ \\
\hline $10-12$ years & $8863(25.2)$ & $1542(23.7)$ & $7321(27.1)$ & 3144 (39.7) & $657(38.0)$ & $2487(40.2)$ \\
\hline$<2$ years tertiary & $11902(33.8)$ & $2178(33.5)$ & 9724 (36.2) & $848(10.7)$ & 203 (11.7) & 645 (10.4) \\
\hline$\geqslant 2$ years tertiary & 10645 (30.2) & 2140 (32.9) & $8505(30.8)$ & $3248(41.0)$ & 742 (42.9) & 2506 (40.5) \\
\hline Missing & $1867(5.3)$ & $292(4.5)$ & $1575(5.5)$ & $322(4.1)$ & 68 (3.9) & $254(4.1)$ \\
\hline Smoking mother & & & & NA & NA & NA \\
\hline No smoking & $26792(76.2)$ & $5093(78.4)$ & $21699(75.6)$ & & & \\
\hline Cigars/pipe & $91(0.3)$ & $17(0.3)$ & $74(0.3)$ & & & \\
\hline$<10$ cigarettes & $3673(10.4)$ & $655(10.1)$ & 3018 (10.5) & & & \\
\hline$>10$ cigarettes & 4170 (11.8) & 635 (9.8) & 3535 (12.3) & & & \\
\hline Do not know & $59(0.2)$ & $9(0.1)$ & $50(0.2)$ & & & \\
\hline Always outside & $83(0.2)$ & $25(0.4)$ & $58(0.2)$ & & & \\
\hline Missing & 315 (0.9) & $63(1.0)$ & 252 (0.9) & & & \\
\hline Smoking father & & & & NA & NA & NA \\
\hline No smoking & $24424(69.4)$ & 4605 (70.9) & 19819 (69.1) & & & \\
\hline Cigars/pipe & $796(2.3)$ & $146(2.2)$ & $650(2.2)$ & & & \\
\hline$<10$ cigarettes & 3624 (10.3) & 641 (9.9) & $2983(10.4)$ & & & \\
\hline >10 cigarettes & 5531 (15.7) & $952(14.6)$ & 4579 (16.0) & & & \\
\hline Do not know & 184 (0.5) & $38(0.6)$ & $146(0.5)$ & & & \\
\hline Always outside & 193 (0.5) & $40(0.6)$ & $153(0.6)$ & & & \\
\hline Missing & 431 (1.2) & $75(1.2)$ & 356 (1.2) & & & \\
\hline
\end{tabular}

Data are presented as $\mathrm{n}(\%)$, unless otherwise stated. IQR: interquartile range; NA: not available. 
TABLE 3 Early-life antibiotic use and subsequent risk of asthma

\begin{tabular}{|c|c|c|c|}
\hline & AB/asthma & $\mathrm{AB} /$ without asthma & Adjusted OR $(95 \% \mathrm{CI})$ \\
\hline \multicolumn{4}{|l|}{ NTR } \\
\hline Unmatched & $2132 / 4896$ (43.5) & $8260 / 24988(33.1)$ & $1.34(1.28-1.41)^{\#, * * *}$ \\
\hline $\mathrm{MZ}$ and same-sex DZ & $534 / 1312(40.7)$ & 482/1312 (36.7) & $1.54(1.20-1.98)^{\uparrow, * *}$ \\
\hline Same-sex DZ & $383 / 924$ (41.5) & $342 / 924(37.0)$ & $1.60(1.18-2.16)^{\uparrow \cdot * *}$ \\
\hline$M Z$ & $151 / 388(38.9)$ & $140 / 388(36.1)$ & $1.52(0.89-2.60)^{\pi}$ \\
\hline \multicolumn{4}{|l|}{ CATSS } \\
\hline Unmatched & $768 / 1381(55.6)$ & $2455 / 5825(42.1)$ & $1.45(1.34-1.56)^{+, * * *}$ \\
\hline MZ and same-sex DZ & $206 / 380(54.2)$ & $174 / 380(45.7)$ & $2.00(1.28-3.13)^{\uparrow, * *}$ \\
\hline Same-sex DZ & $141 / 275$ (51.3) & $122 / 275(44.4)$ & $1.86(1.11-3.18)^{\uparrow 1, *}$ \\
\hline $\mathrm{MZ}$ & $65 / 105(61.9)$ & $55 / 105(52.4)$ & $2.23(0.90-5.53)^{\pi}$ \\
\hline \multicolumn{4}{|c|}{$\begin{array}{l}\text { Data are presented as } \mathrm{n} / \mathrm{n}(\%) \text {, unless otherwise stated. AB: users of any antibiotics; NTR: Netherlands } \\
\text { Twin Registry; MZ: monozygotic twin pair level; DZ: dizygotic twin pair level; CATSS: Childhood and } \\
\text { Adolescent Twin Study in Sweden. \#: adjusted for socioeconomic status, hours of outside childcare, } \\
\text { breastfeeding, delivery mode, sex and birthweight; }{ }^{\uparrow} \text { : adjusted for birthweight; }{ }^{+} \text {: adjusted for educational } \\
\text { attainment, delivery mode, sex and birthweight. }{ }^{*}: p<0.05 ;{ }^{* *}: p<0.01{ }^{* * *}: p<0.001 .\end{array}$} \\
\hline
\end{tabular}

antibiotics (narrow-spectrum antibiotics OR 1.83, 95\% CI 1.50-2.24; broad-spectrum antibiotics OR 1.24, 95\% CI 1.11-1.38). For narrow-spectrum antibiotics, the direction of the association remained after controlling for shared familial environment factors (OR 1.95, 95\% CI 1.22-3.14) when studying monozygotic and dizygotic twins and when exposure to broad-spectrum antibiotics was studied (OR 1.38, 95\% CI 0.63-3.03), with some loss in precision. Point estimates were similar, but confidence intervals included the null when restricted to monozygotic twins for use of narrow-spectrum antibiotics (OR 1.92, 95\% CI 0.73-4.99) and for use of broad-spectrum antibiotics (OR 3.13, 95\% CI 0.79-12.47) (supplementary tables S7a and b).

\section{Antibiotics and eczema}

Increased risk of eczema was observed after early-life antibiotic use in the unmatched cohort analysis in the NTR (OR 1.08, 95\% CI 1.03-1.13) and the CATSS (OR 1.19, 95\% CI 1.03-1.38) (table 5). However, this risk attenuated after controlling for shared familial environment factors in the NTR including same-sex dizygotic pairs (OR 0.99, 95\% CI 0.80-1.25), but not in the CATSS (OR 1.67, 95\% CI 1.12-2.49). In monozygotic pairs, the association attenuated in the NTR (OR 1.02, 95\% CI 0.69-1.52), but increased odds were found in the CATSS (OR 1.76, 95\% CI 0.98-3.14).

\section{Sensitivity analyses for eczema}

The results of our sensitivity analyses in which we 1) only included eczema in those aged over 3 years and 2) re-defined eczema based on medication and diagnosis (CATSS) showed consistent results in the NTR.

TABLE 4 Early-life antibiotic use commonly prescribed for urinary tract/skin infections and subsequent risk of asthma (with correction for respiratory antibiotics)

\section{AB/asthma}

$72 / 1314(5.5)$

$16 / 380(4.2)$

$12 / 275(4.4)$

$4 / 105(3.8)$
$A B /$ without asthma

$263 / 5584(4.7)$
$18 / 380(4.7)$
$13 / 275(4.7)$
$5 / 105(4.8)$

Adjusted OR $(95 \% \mathrm{Cl})$

\section{CATSS}

$\mathrm{MZ}$ and same-sex DZ

Same-sex DZ

$M Z$

)

Data are presented as $n / n(\%)$, unless otherwise stated. $A B$ : users of antibiotics commonly prescribed for urinary tract/skin infections (pivmecillinam, trimethoprim, sulphonamide, ciprofloxacin, norfloxacin, nitrofurantoin, cloxacillin, flucloxacillin and dicloxacillin); CATSS: Childhood and Adolescent Twin Study in Sweden; MZ: monozygotic twin pair level; DZ: dizygotic twin pair level. \#: adjusted for: antibiotics commonly prescribed for respiratory infections (amoxicillin, penicillin, cephalosporin and macrolides), educational attainment, delivery mode, sex and birthweight; ": adjusted for respiratory antibiotics and birthweight. 


\begin{tabular}{|c|c|c|c|}
\hline & AB/eczema & $A B /$ without eczema & Adjusted OR $(95 \% \mathrm{CI})$ \\
\hline \multicolumn{4}{|l|}{ NTR } \\
\hline Unmatched & $2286 / 5918$ (38.6) & 7763/22983 (33.8) & $1.08(1.03-1.13)^{\#}$ \\
\hline MZ and same-sex DZ & $687 / 1837(37.4)$ & $684 / 1837(37.2)$ & $0.99(0.80-1.25)^{9}$ \\
\hline Same-sex DZ & $441 / 1191(37.0)$ & $441 / 1191$ (37.0) & $0.96(0.73-1.26)^{9}$ \\
\hline$M Z$ & $243 / 646(37.6)$ & $243 / 646(37.6)$ & $1.02(0.69-1.52)^{\pi}$ \\
\hline \multicolumn{4}{|l|}{ CATSS } \\
\hline Unmatched & $819 / 1730(47.3)$ & $2278 / 5114$ (44.5) & $1.19(1.03-1.38)^{+, *}$ \\
\hline $\mathrm{MZ}$ and same-sex DZ & $296 / 624(47.4)$ & $255 / 624(40.9)$ & $1.67(1.12-2.49)^{\Upsilon 1, *}$ \\
\hline Same-sex DZ & $187 / 411$ (45.5) & $160 / 411(38.9)$ & $1.71(1.10-2.68)^{\uparrow 1, *}$ \\
\hline$M Z$ & $109 / 213(51.2)$ & $95 / 213(44.6)$ & $1.76(0.98-3.14)^{9}$ \\
\hline \multicolumn{4}{|c|}{$\begin{array}{l}\text { Data are presented as } \mathrm{n} / \mathrm{n}(\%), \text { unless otherwise stated. AB: users of any antibiotics; NTR: Netherlands } \\
\text { Twin Registry; MZ: monozygotic twin pair level; DZ: dizygotic twin pair level; CATSS: Childhood and } \\
\text { Adolescent Twin Study in Sweden. "\#: adjusted for socioeconomic status, hours of outside childcare, } \\
\text { breastfeeding, delivery mode, sex, birthweight and asthma; } \text { १: adjusted for birthweight and asthma; }^{+} \text {: adjusted for educational attainment, delivery mode, sex, birthweight and asthma. *: p<0.05. }\end{array}$} \\
\hline
\end{tabular}

In the CATSS the risk estimates for same-sex dizygotic and monozygotic pairs moved towards the null compared with unmatched analyses (supplementary tables S8 and S9).

\section{Discussion}

In this large twin study, early-life exposure to antibiotics was associated with the development of asthma, even after correcting for shared familial environment factors in same-sex dizygotic discordant twin pairs. After correction for shared familial environment and genetic factors in monozygotic discordant twin pairs, there was reduced power, but the point estimates were in the same direction. The association between early-life antibiotic exposure and risk of eczema was potentially confounded by shared familial environment and genetic factors.

This study is the first using a co-twin control analysis assessing the impact of confounding by shared familial environment and genetic factors in the association of early-life antibiotic use with asthma and eczema. Our finding that early-life exposure to antibiotics was associated with an increased risk of asthma in the unmatched cohort study in the NTR and the CATSS aligns with previous population-based cohort studies suggesting that populations of twins may be representative of the general paediatric population [12, $16,21]$. Note that the overall rate of antibiotic exposure in our population is consistent with national data describing antibiotic use in young children in the Netherlands (31\% in 2019) and Sweden (26-61\% depending on the region in 2010); these percentages tend to be among the lowest in the world [30, 31].

In our study, after analysing matched discordant twins (both monozygotic and dizygotic) analyses, the risk of asthma remained for antibiotics commonly prescribed for respiratory infections, but not for antibiotics commonly prescribed for urinary tract/skin infections. These results correspond with findings in the Swedish sibling study: increased risk of asthma was observed in siblings treated with antibiotics commonly prescribed for respiratory tract infections in early life, but not for urinary tract/skin infections [12]. In contrast, a Finnish nested case-control study ( $\mathrm{n}=6690$ case-control pairs) found an increase in risk (OR $1.60,95 \%$ CI 1.48-1.73) of asthma in 23 children treated with antibiotics commonly prescribed for urinary tract infections (defined as trimethoprim and its combinations with sulphonamides) in early life, which differed from our definition of antibiotics prescribed for urinary tract/skin infections [32]. A relatively small sample size and differences in selected antibiotics may explain this discordance in findings.

Our study also shows that narrow-spectrum and broad-spectrum antibiotics use was associated with increased risk of asthma, even after controlling for shared familial environment and genetic factors, which was consistent with other studies. A birth cohort study of 13116 Canadian children found increased risk of asthma at age 7 years for children exposed during the first year of life to broad-spectrum antibiotics (OR 1.50, 95\% CI 1.16-1.93) and narrow-spectrum antibiotics (OR 1.35, 95\% CI 0.29-6.23), although the broad-spectrum and narrow-spectrum study suffered from reduced power [33]. Moreover, a Finnish nested case-control study also showed a positive association in 41 children treated with antibiotics against Gram-positive bacterial infections and urinary tract infections, overlapping with the broad-spectrum antibiotics in our analysis [32]. However, to the best of our knowledge, we are the first to investigate the effects of antibiotic spectra in a twin design. Prescription of narrow-spectrum antibiotics was more 
common in our population compared with broad-spectrum antibiotics in both the group with and the group without asthma, when adjusted for overlap of use of both types of antibiotics groups within the time frame of 2 years. We would have expected to observe a similar effect of narrow-spectrum and broad-spectrum antibiotics, or broad-spectrum antibiotics should have even shown a larger risk of asthma, regarding the hypothesis of its influence on the microbiota. If the respiratory infection is driving the asthma, this could be a potential explanation. However, we lack information regarding respiratory infections and cannot conclude this with the current data.

In the NTR, the association between early-life antibiotic use and eczema attenuated in the co-twin control analysis, suggesting possible confounding by shared familial environment and genetic factors. In the CATSS, the association between early-life antibiotic use and eczema also attenuated when the definition for eczema was based on medication and diagnoses after 2 years, rather than parental report for ever-eczema. Since eczema is common in children aged under 2 years, the parental-report definition is open to reverse causation bias, and therefore the validated definition using medication and diagnosis in those aged over 2 years is a more robust measure. Our results in the unmatched analyses are similar to previous studies, which did not assess confounding by shared genetic or familial factors [17, 20]. Considering the aforementioned, we hypothesise that the association between early-life antibiotic use and eczema may be driven by confounding from shared environmental factors, whereas this may not be the case for asthma. We expected to find similar findings for asthma and eczema as the causal pathways of both diseases are assumed to be similar. A large genome-wide association study of a multidisease phenotype based on information from three genetically correlated diseases (asthma, hay fever and eczema) also identified six variants with disease-specific effects, besides 130 overlapping variants [34], showing that the three diseases share some but not all characteristics. Confounding factors may also be disease specific and cannot be unravelled in the current study design.

The positive association between all antibiotics and risk of asthma can possibly be elucidated by the microbial diversity hypothesis. It states that early-life exposure to microorganisms increases variety in gut microflora and type 1 immune system development, and therefore protects against asthma [35, 36]. Antibiotic therapy affects microbiome variety and thus may increase the risk of atopic diseases. Another possible underlying reason may be that the associations observed were due to the severity of underlying infections rather than the antibiotic itself. Respiratory viral infections, in particular, have been shown to initiate a cascade of host immune responses altering microbial growth in the respiratory tract and gut [37]. In our analysis, we attempted to assess the impact of confounding by indication due to respiratory infections using antibiotic subgroups as proxies for infections (respiratory versus urinary tract/skin infections). The analysis for urinary tract infection antibiotics showed no associations and therefore could suggest that the association between antibiotics for respiratory infections and asthma is due to confounding by indication, but there are some limitations to this analysis. Infections that do not warrant antibiotics are not captured in our databases and, as such, we could not assess whether observed associations were due to specific infections or antibiotics, as they occur simultaneously. Moreover, the small sample size in the urinary tract infection subgroup may not have allowed us to detect moderate associations in this subgroup. Therefore, we hesitate to conclude that confounding by indication is driving the results.

The strength of our study is the large discovery cohort including almost 35000 Dutch twins and replication in almost 8000 Swedish twins. We found similar results for asthma in the CATSS compared with the NTR, meaning that our results are generalisable to Western European children. Our twin design controls for shared familial environment and genetic factors. We addressed recall bias, reverse causation for asthma, and validated our asthma and eczema definition using International Classification of Diseases codes.

This study also had some limitations. First, our discovery cohort only consisted of parental-reported antibiotic use. Nevertheless, antibiotic use between 0 and 2 years was ascertained in the NTR at age 2 years, minimising recall bias. Moreover, after defining use of antibiotics with medication prescription claims in the CATSS, results were similar. Second, our subgroup analyses had reduced power because ATC codes were only available in the CATSS (supplementary material). Third, outcome misclassification may have occurred due to parental-reported measures for asthma and eczema. Fourth, more children in the CATSS were delivered via caesarean section compared with the NTR and this may result in differences in risks of asthma or eczema development.

To conclude, despite familial and genetic factors, children exposed to early-life antibiotic use, particularly antibiotics commonly prescribed for respiratory tract infections, may be at a higher risk of asthma. Antibiotic use was also associated with increased risk of eczema; however, it is likely that the relationship between early-life antibiotic use and eczema is confounded by shared familial environment and genetic factors. 
Given our findings, physicians should ensure that antibiotics are prescribed for bacterial infections only. Future studies should have a prospective design monitoring timing of events, type of infection and number of antibiotics used in early life.

Acknowledgements: We acknowledge the Netherlands Twin Registry and the Swedish Twin Registry for access to data.

Conflict of interest: E.M.A. Slob has nothing to disclose. B.K. Brew has nothing to disclose. S.J.H. Vijverberg has nothing to disclose. C.J.A.R. Kats has nothing to disclose. C. Longo has nothing to disclose. M.W. Pijnenburg has nothing to disclose. T.C.E.M. van Beijsterveldt has nothing to disclose. C.V. Dolan has nothing to disclose. M. Bartels has nothing to disclose. P. Magnusson has nothing to disclose. P. Lichtenstein has nothing to disclose. T. Gong has nothing to disclose. G.H. Koppelman reports grants from Lung Foundation of the Netherlands, Teva, Ubbo Emmius Foundation, Tetri Foundation, GSK and Vertex, outside the submitted work; and has participated in a global advisory board on paediatric asthma for GSK. C. Almqvist has nothing to disclose. D.I. Boomsma has nothing to disclose. A.H. Maitland-van der Zee reports grants and personal fees for advisory board work from GSK and Boehringer Ingelheim, personal fees for advisory board work from AstraZeneca, grants from Chiesi, outside the submitted work.

Support statement: E.M.A. Slob was supported by the Dutch Lung Foundation, Amsterdam Public Health, AMC Young Talent Fund and Jo Kolk Studiefonds. The Netherlands Twin Registry gratefully acknowledges NWO-Groot (grant 480-15-001/674: Netherlands Twin Registry Repository) and the Royal Netherlands Academy of Science Professor Award (PAH/6635) to D.I. Boomsma. Financial support for the Childhood and Adolescent Twin Study in Sweden was provided from the Swedish Research Council (framework grant 340-2013-5867), grants from the Stockholm County Council (ALF-projects) and the Swedish Heart-Lung Foundation. The Swedish Twin Registry is managed by Karolinska Institutet and receives funding through the Swedish Research Council (grant 2017-00641). Funding information for this article has been deposited with the Crossref Funder Registry.

\section{References}

1 Thomsen SF. Epidemiology and natural history of atopic diseases. Eur Clin Respir J 2015; 2: 24642.

2 O'Connell EJ. The burden of atopy and asthma in children. Allergy 2004; 59: Suppl. 78, 7-11.

3 Otters HB, van der Wouden JC, Schellevis FG, et al. Trends in prescribing antibiotics for children in Dutch general practice. J Antimicrob Chemother 2004; 53: 361-366.

4 Dekker ARJ, Verheij TJM, van der Velden AW. Antibiotic management of children with infectious diseases in Dutch Primary Care. Fam Pract 2017; 34: 169-174.

5 Kuo $\mathrm{CH}$, Kuo HF, Huang $\mathrm{CH}$, et al. Early life exposure to antibiotics and the risk of childhood allergic diseases: an update from the perspective of the hygiene hypothesis. J Microbiol Immunol Infect 2013; 46: 320-329.

6 Bloomfield SF, Rook GA, Scott EA, et al. Time to abandon the hygiene hypothesis: new perspectives on allergic disease, the human microbiome, infectious disease prevention and the role of targeted hygiene. Perspect Public Health 2016; 136: 213-224.

7 Björkstén B, Sepp E, Julge K, et al. Allergy development and the intestinal microflora during the first year of life. J Allergy Clin Immunol 2001; 108: 516-520.

8 Kalliomäki M, Kirjavainen P, Eerola E, et al. Distinct patterns of neonatal gut microflora in infants in whom atopy was and was not developing. J Allergy Clin Immunol 2001; 107: 129-134.

9 Penders J, Thijs C, Vink C, et al. Factors influencing the composition of the intestinal microbiota in early infancy. Pediatrics 2006; 118: 511-521.

10 Dharmage SC, Lodge CJ, Lowe AJ, et al. Antibiotics and risk of asthma: a debate that is set to continue. Clin Exp Allergy 2015; 45: 6-8.

11 Homaira N, Briggs N, Pardy C, et al. Association between respiratory syncytial viral disease and the subsequent risk of the first episode of severe asthma in different subgroups of high-risk Australian children: a whole-of-population-based cohort study. BMJ Open 2017; 7: e017936.

12 Örtqvist AK, Lundholm C, Kieler H, et al. Antibiotics in fetal and early life and subsequent childhood asthma: nationwide population based study with sibling analysis. BMJ 2014; 349: g6979.

13 Kuehni CE, Spycher BD, Silverman M. Causal links between RSV infection and asthma: no clear answers to an old question. Am J Respir Crit Care Med 2009; 179: 1079-1080.

14 Scheltema NM, Nibbelke EE, Pouw J, et al. Respiratory syncytial virus prevention and asthma in healthy preterm infants: a randomised controlled trial. Lancet Respir Med 2018; 6: 257-264.

15 von Mutius E. Allergies, infections and the hygiene hypothesis - the epidemiological evidence. Immunobiology 2007; 212: 433-439.

16 Metzler S, Frei R, Schmaußer-Hechfellner E, et al. Association between antibiotic treatment during pregnancy and infancy and the development of allergic diseases. Pediatr Allergy Immunol 2019; 30: 423-433.

17 Tsakok T, McKeever TM, Yeo L, et al. Does early life exposure to antibiotics increase the risk of eczema? A systematic review. Br J Dermatol 2013; 169: 983-991.

18 Cullinan P, Harris J, Mills P, et al. Early prescriptions of antibiotics and the risk of allergic disease in adults: a cohort study. Thorax 2004; 59: 11-15.

19 Dom S, Droste JH, Sariachvili MA, et al. Pre- and post-natal exposure to antibiotics and the development of eczema, recurrent wheezing and atopic sensitization in children up to the age of 4 years. Clin Exp Allergy 2010; 40: 1378-1387.

20 Ahmadizar F, Vijverberg SJH, Arets HGM, et al. Early-life antibiotic exposure increases the risk of developing allergic symptoms later in life: a meta-analysis. Allergy 2018; 73: 971-986.

21 Ahmadizar F, Vijverberg SJH, Arets HGM, et al. Early life antibiotic use and the risk of asthma and asthma exacerbations in children. Pediatr Allergy Immunol 2017; 28: 430-437.

22 Huang FQ, Lu CY, Wu SP, et al. Maternal exposure to antibiotics increases the risk of infant eczema before one year of life: a meta-analysis of observational studies. World J Pediatr 2020; 16: 143-151.

23 Lichtenstein P, De Faire U, Floderus B, et al. The Swedish Twin registry: a unique resource for clinical, epidemiological and genetic studies. J Intern Med 2002; 252: 184-205. 
24 van Beijsterveldt CE, Groen-Blokhuis M, Hottenga JJ, et al. The Young Netherlands Twin Register (YNTR): longitudinal twin and family studies in over 70,000 children. Twin Res Hum Genet 2013; 16: 252-267.

25 Anckarsäter H, Lundström S, Kollberg L, et al. The Child and Adolescent Twin Study in Sweden (CATSS). Twin Res Hum Genet 2011; 14: 495-508.

26 Ludvigsson JF, Almqvist C, Bonamy AK, et al. Registers of the Swedish total population and their use in medical research. Eur J Epidemiol 2016; 31: 125-136.

27 Clausen TD, Bergholt T, Bouaziz O, et al. Broad-spectrum antibiotic treatment and subsequent childhood type 1 diabetes: a nationwide Danish cohort study. PLoS One 2016; 11: e0161654.

28 Demidenko E. Sample size determination for logistic regression revisited. Stat Med 2007; 26: 3385-3397.

29 Demidenko E. Sample size and optimal design for logistic regression with binary interaction. Stat Med 2008; 27: 36-46.

30 Stichting Farmaceutische Kengetallen. Jonge kinderen vooral lokaal werkende geneesmiddelen. [Young children mostly local acting medicines.] Pharm Weekbl 2019; 154: 38.

31 Swedish Institute for Communicable Disease Control. A Report on Swedish Antibiotic Utilisation and Resistance in Human Medicine. Solna, Swedish Institute for Communicable Disease Control, 2010.

32 Metsala J, Lundqvis A, Virta L, et al. Prenatal and post-natal exposure to antibiotics and risk of asthma in childhood. Clin Exp Allergy 2014; 45: 137-145.

33 Kozyrskyj AL, Ernst P, Becker AB. Increased risk of childhood asthma from antibiotic use in early life. Chest 2007; 131: 1753-1759.

34 Ferreira MA, Vonk JM, Baurecht $\mathrm{H}$, et al. Shared genetic origin of asthma, hay fever and eczema elucidates allergic disease biology. Nat Genet 2017; 49: 1752-1757.

35 Strachan DP. Hay fever, hygiene, and household size. BMJ 1989; 299: 1259-1260.

36 von Mutius E. The microbial environment and its influence on asthma prevention in early life. J Allergy Clin Immunol 2016; 137: 680-689.

37 Hanada S, Pirzadeh M, Carver KY, et al. Respiratory viral infection-induced microbiome alterations and secondary bacterial pneumonia. Front Immunol 2018; 9: 2640 